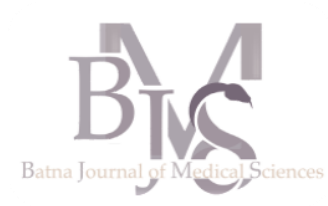

Faculté de Médecine, Université Alger 1, Alger - Algérie

\section{Correspondance à :}

Fadia RAHAL

ffadia 2007@yahoo.fr

DOI :https://doi.org/10.48087/ BJMStf.2015.2115

Il s'agit d'un article en libre accès distribué selon les termes de la licence Creative Commons Attribution International License (CC BY 4.0), qui autorise une utilisation, une distribution et une reproduction sans restriction sur tout support ou format, à condition que l'auteur original et la revue soient dûment crédités.

\title{
Maladie de Gaucher
}

\section{Gaucher's disease}

\section{Fadia Rahal, Nadjia Brahimi, Aicha Ladjouze-Rezig}

\section{RÉSUMÉ}

La maladie de Gaucher (MG) est une maladie lysosomale due au déficit génétique en bglucosidase, de transmission autosomique récessive; on en distingue trois types. La MG de type 1 , la plus fréquente, se caractérise par une splénomégalie dans $90 \%$ des cas, une cytopénie (essentiellement, anémie et thrombopénie), et une atteinte ostéoarticulaire infiltrative et destructrice qui impacte souvent la qualité de vie des patients. Les deux autres phénotypes incluent une atteinte neurologique, sévère dans le type 2 qui affecte le nourrisson et évolue rapidement vers le décès, et plus lentement progressive dans le type 3 qui comporte également les symptômes du type 1 . Le diagnostic repose sur le dosage de la bglucosidase dans les leucocytes. Cependant, la maladie de Gaucher reste peu connue, et son diagnostic est souvent fait tardivement particulièrement en milieu rhumatologique ou se sont essentiellement les différentes complications ostéarticulaires associées aux manifestations hématologiques qui permettent d'orienter vers cette maladie. Par ailleurs, l'approche thérapeutique dominante est l'enzymothérapie substitutive (ETS), disponible actuellement pour certaines maladies lysosomales.

Mots clés : maladie lysosomale, hépatosplénomegalie, ostéonécrose aseptique, enzymothérapie substitutive

\begin{abstract}
Gaucher's disease (GD) is a lysosomal disease which is due to a genetic defect in bglucosidase, with autosomal recessive transmission; there are 3 types. Type $1 \mathrm{GD}$ is the most frequent and is characterized by splenomegaly in $90 \%$ of cases, cytopenia (mainly anemia and throbopenia) and a musculoskeletal involvement, with infiltration and destruction that can compromise the patient's life. The two other phenotypes include a severe neurological involvement in type 2 that affects infants and is deadly; in type 3 the neurological involvement is less severe and is also associated with features of types 1 . Diagnosis is made based on the dosing of the bglucosidase in leucocytes. However, GD is still not very well known and its diagnosis is made very late, in rheumatological setting in the context of musculoskeletal complications associated with hematological involvement, which allow to guide the diagnosis. Furthermore, the main therapeutic approach includes enzyme replacement therapies, available in some lysosomal diseases.
\end{abstract}

Keywords: Lysosomal disease, hepatoplenomegaly, aseptic necrosis, enzyme replacement therapy.

\section{Pour citer l'article :}

Rahal F, N Brahimi, A Ladjouze-Rezig. Maladie de Gaucher. Batna J Med Sci 2015;2(1):66-69. https://doi.org/10.48087/ BJMStf.2015.2115

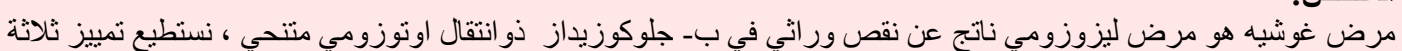

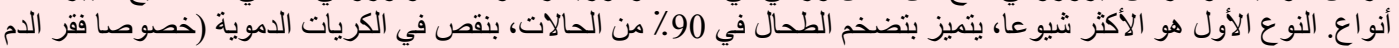

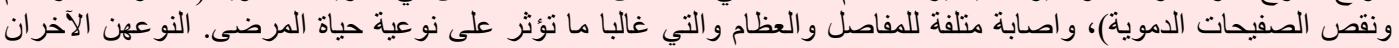

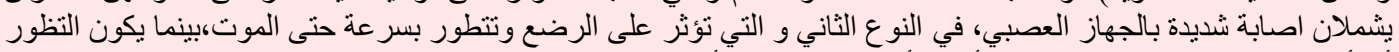

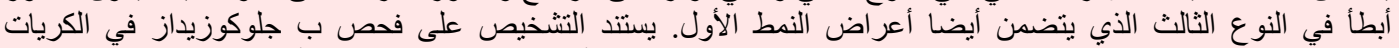

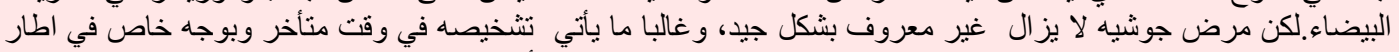

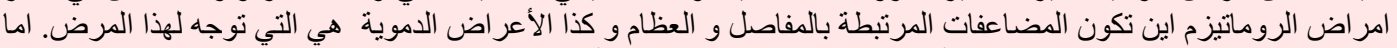

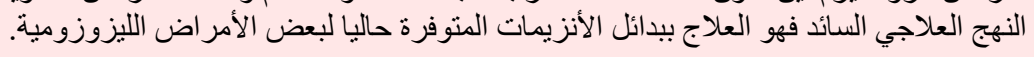
كلمات البحث: المرض الليزوزومي، ضخامة الكبد والطحال، تنخر العظم، العلاج ببدائل الأنزيمات 


\section{INTRODUCTION}

La maladie de Gaucher (MG) est une affection génétique rare, autosomique récessive, due à un déficit de l'activité de l'enzyme lysosomale : la glucocérébrosidase.

Considérée comme la plus fréquente des maladies lysosomales, la MG est héréditaire à transmission autosomique récessive et sa distribution est ubiquitaire [1], elle se caractérise par une hépatosplénomégalie fréquente, une atteinte osseuse parfois sévère et, dans certaines formes rares, une atteinte neurologique [2]. La prévalence de la MG est de l'ordre de 1/60 000 dans la population générale, mais peut atteindre 1/1000 dans la population juive ashkénaze.

\section{PHYSIOPATHOLOGIE}

La glucocérébrosidase est une enzyme lysosomale qui hydrolyse le glucosylcéramide (lipide complexe) en céramide et glucose (figure 1). Dans la maladie de Gaucher, le glucosylcéramide non dégradé s'accumule dans les lysosomes des cellules du système réticulo-endothélial (macrophages), leur donnant un aspect caractéristique en microscopie " cytoplasme en aspect de papier froissé et noyau excentré » (figure 2). Ces macrophages, appelés cellules de Gaucher, vont infiltrer le foie, la rate et la moelle osseuse, et sont responsables des principaux signes cliniques de la maladie [3].

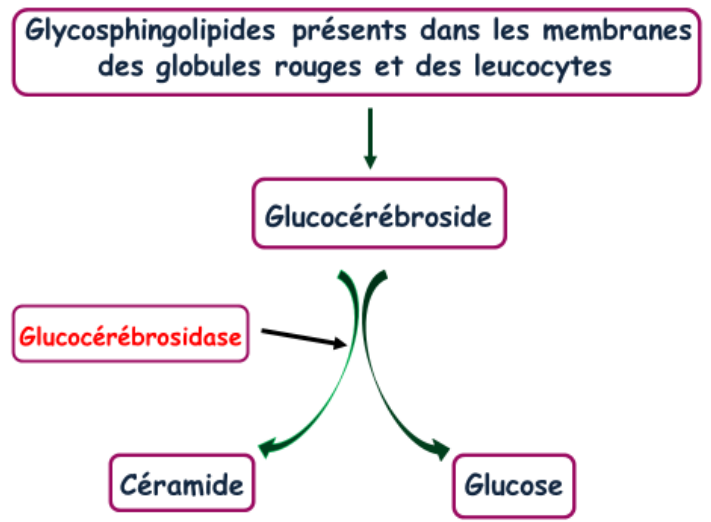

Figure 1 : catabolisme du glucocérébroside

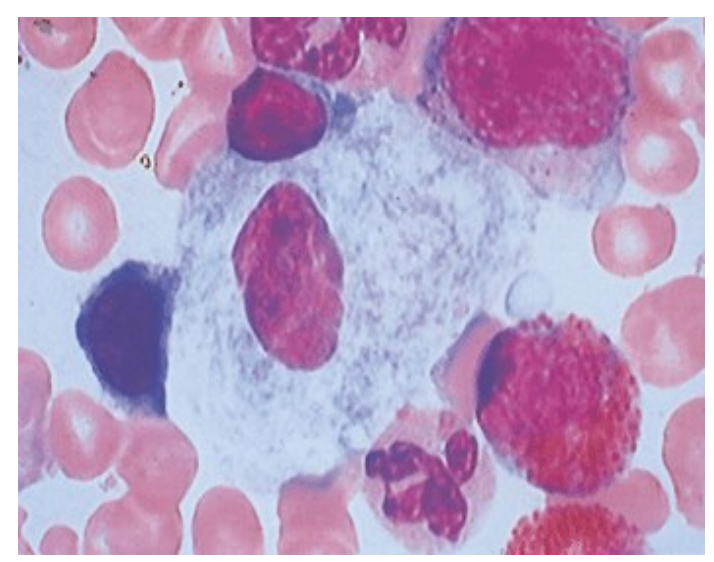

Figure 2 : cellule de Gaucher

\section{DESCRIPTION CLINIQUE}

On décrit classiquement trois phénotypes définis sur des caractéristiques cliniques.

\section{Type 1, sans atteinte neurologique}

Le type 1, défini classiquement par l'absence d'atteinte neurologique [4], est la forme la plus fréquente (95\%). La présentation clinique est très hétérogène, avec parfois des formes asymptomatiques. L'asthénie est fréquente retentissant souvent sur la vie scolaire et socioprofessionnelle. La splénomégalie, parfois majeure, peut aboutir à un véritable infarctus splénique; elle est présente chez $90 \%$ des patients. L'hépatomégalie est notée chez plus de $80 \%$ des patients.

L'hépatosplénomégalie entraîne parfois une distension abdominale douloureuse qui peut comprimer les autres organes et gêner la respiration. L'échographie, et si possible, l'imagerie par résonance magnétique (IRM) abdominale, est un élément important de l'évaluation initiale et de la surveillance.

L'atteinte osseuse est présente dans $80 \%$ des cas et peut retentir sur le pronostic fonctionnel : troubles du remodelage osseux, ostéopénie, ostéoporose, fractures pathologiques et tassements vertébraux (figure 3), lyses osseuses, infarctus osseux se manifestant par des crises douloureuses intenses et invalidantes; ostéonécroses aseptiques (têtes fémorales, têtes humérales, etc.) pouvant évoluer vers une arthropathie dégénérative (figure 4) et justifiant la mise en place d'une prothèse.
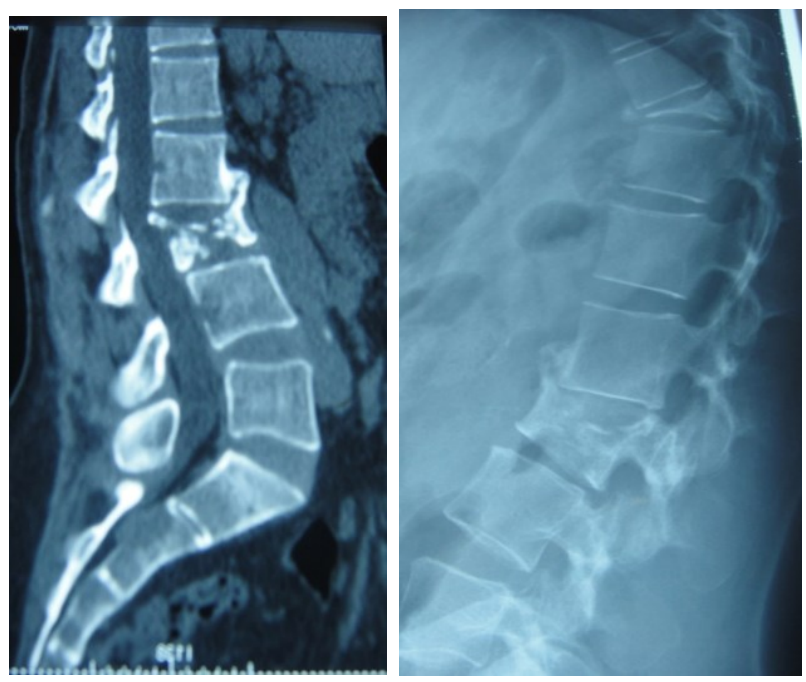

Figure 3 : fractures vertebrales : D11 et L3

L'atteinte des autres organes est rare : atteinte pulmonaire (atteinte interstitielle, fibrose pulmonaire, syndrome restrictif secondaire aux déformations du rachis, hypertension artérielle pulmonaire); atteinte cardiaque (infiltration myocardique ou péricardique, calcifications valvulaires rapportées essentiellement dans le type 3); atteinte rénale se manifestant par une protéinurie et une hématurie, traduisant les dépôts de cellules de Gaucher dans les glomérules ; exceptionnelles atteintes cutanée, oculaire ou digestive.

\section{Type 2, aigu neuronopathique}

Le type $2(1 \%)$, caractérisé par une atteinte neurologique précoce et sévère [5], débute généralement chez le 
nourrisson de trois à six mois par une atteinte systémique avec hépatosplénomégalie et manifestations neurologiques gravissimes.

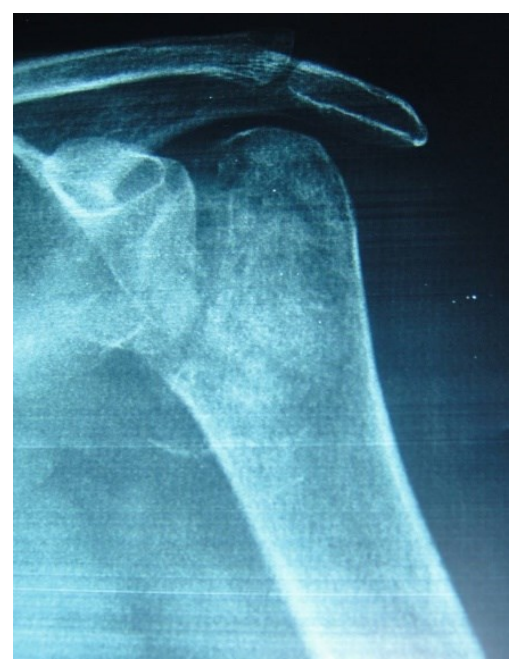

Figure 4 : ostéonécrose de la tête humérale

Les premiers signes sont une paralysie oculomotrice ou un strabisme fixé bilatéral associés secondairement à des signes bulbaires, en particulier des troubles sévères de la déglutition, une spasticité progressive et des mouvements dystoniques. Les convulsions sont plus tardives, se manifestant par une épilepsie myoclonique résistante aux traitements antiépileptiques. Le décès survient le plus souvent avant l'âge de deux ans. Le traitement enzymatique est inefficace.

\section{Type 3}

Le type $3(5 \%)$ est caractérisé par une atteinte neurologique d'apparition plus tardive et d'évolution plus progressive que dans le type 2 [6]. Il est également appelé type juvénile ou type neurologique subaigu. Comme pour le type 1 , il regroupe des malades très hétérogènes. Certains patients ont une atteinte systémique modérée et une ophtalmoplégie associée, comme seul symptôme neurologique. Pour les formes plus sévères les signes neurologiques rencontrés sont variables : ophtalmoplégie horizontale supra nucléaire, épilepsie myoclonique progressive, ataxie cérébelleuse, spasticité et démence dans certains cas. Le traitement enzymatique semble ralentir l'évolution de l'atteinte neurologique. Sans traitement spécifique, l'évolution se fait vers le décès en quelques années.

\section{EXAMENS COMPLÉMENTAIRES POUR DIAGNOSTIC DE LA MALADIE DE GAUCHER}

\section{Myélogramme ou biopsie ostéomédullaire}

Ils permettent de mettre en évidence des cellules de Gaucher, qui sont de grosses cellules macrophagiques dont le cytoplasme est plissé et qui contiennent des inclusions lysosomales constituées de glucosylcéramide. Les cellules de Gaucher ne sont pas spécifiques de la maladie. En effet, on peut retrouver des cellules avec les mêmes caractéristiques cytologiques ("pseudo-Gaucher») dans la leucémie myéloïde chronique, au cours du myélome ou d'autres hémopathies. Le myélogramme permet de caractériser le type de cytopénie.

\section{Dosage de la bêta-glucocérébrosidase}

C'est le dosage de l'activité enzymatique déficiente, il permet de faire le diagnostic. Les valeurs de l'activité enzymatique sont de 10 à $30 \%$ des valeurs normales, parfois moins. Le taux n'est pas prédictif de la sévérité de la maladie.

\section{Recherche de mutations}

On peut rechercher les cinq mutations les plus fréquentes par Polymerase Chain Reaction (PCR). Elles sont parfois un lien avec l'expression clinique de la maladie.

\section{Marqueurs biologiques}

La pancytopénie (par hypersplénisme et/ou infiltration médullaire) associe à des degrés divers une thrombopénie habituelle et rarement profonde, une anémie et une leuconeutropénie (plus rare et peu sévère), Le myélogramme, outre les cellules de Gaucher qu'il peut mettre en évidence, permet d'en préciser le mécanisme soit central par infiltration médullaire, soit périphérique par destruction ou séquestration splénique [7]. L'anémie est souvent au second plan. Les neutropénies sont parfois sévères.

Il existe parfois des troubles de la coagulation à type d'allongement du TCA, sans déficit en facteur de coagulation, dû à l'interférence des sphingolipides avec les protéines de la coagulation ou à une diminution de facteurs de coagulation par consommation par les macrophages activés. Les accidents hémorragiques sont rares en dehors des thrombopénies sévères.

Signes de l'activation macrophagique: Il existe une augmentation de protéines lysosomales comme LAMP-1 (lysosomalassociated protein) et SAP-C (saposine C) dans les formes sévères de la MG.

Actuellement, les deux marqueurs les plus intéressants pour le suivi de la maladie sont la chitotriosidase est le marqueur le plus intéressant, particulièrement pour le suivi du traitement enzymatique. Sa corrélation avec la sévérité de la maladie est bonne quoique imparfaite; un autre marqueur peut être indiqué pour le suivi de la maladie : La chémokine CCL18 est un marqueur intéressant car il est très spécifique de la MG. Son taux peut atteindre jusqu'à 30 fois la normale. C'est un bon marqueur de suivi thérapeutique qui pourrait être dosé chez les patients sans activité chitotriosidase. Cependant ce dosage n'est pas disponible en pratique courante.

\section{Imagerie de l'appareil locomoteur}

\section{Radiographie conventionnelle}

Elle permet d'évoquer le diagnostic, mais les images radiographiques peuvent prêter à confusion avec les hémoglobinopathies et notamment la drépanocytose ou des autres maladies de surcharges (ostéonécrose, ischémie médullaire...).

Scintigraphie osseuse au 99mTechnétium

Non spécifique, elle sert à établir une cartographie des différentes localisations osseuses de la maladie.

\section{Tomodensitométrie}

Elle peut avoir un intérêt pour préciser une atteinte articulaire ou un envahissement des parties molles en cas d'infection.

\section{Imagerie par résonance magnétique (IRM)}

C'est le moyen d'investigation de choix, elle visualise précocement les complications de la maladie : infarctus osseux, ostéonécrose, lésions infectieuses, lésions des parties molles.

\section{PRISE EN CHARGE}

Les objectifs du traitement de la MG de l'adulte sont triples : 
$\checkmark$ Apporter un soulagement fonctionnel;

$\checkmark$ Améliorer la qualité de vie des patients ;

$\checkmark$ Prévenir les complications.

En matière d'atteinte ostéarticulaire, les objectifs thérapeutiques sont précis :

$\checkmark$ Diminuer ou supprimer les douleurs osseuses dans un délai de un à deux ans ;

$\checkmark$ Prévenir les crises douloureuses osseuses;

$\checkmark$ Prévenir les ostéonécroses et les fractures souschondrales ;

$\checkmark$ Augmenter la DMO trabéculaire dans un délai de trois à cinq ans.

\section{Traitement spécifique}

Actuellement, deux approches thérapeutiques spécifiques sont à notre disposition. Le traitement spécifique n'est pas justifié chez tous les patients. Lorsqu'il est commencé, le traitement doit cependant être poursuivi à vie.

Le traitement de première intention de la maladie de Gaucher de type 1 symptomatique et de la maladie de Gaucher de type 3 est l'enzymothérapie de substitution (8), qui permet la dégradation du glycosylcéramide accumulé, améliorant ainsi les anomalies hématologiques, l'hépatosplénomégalie et la qualité de vie en quelques mois. Deux enzymes ont actuellement obtenu l'autorisation de mise sur le marché (AMM): imiglucérase (Cerezyme) et vélaglucérase (VPRIV). Les enzymes sont dispensés en perfusions tous les 15 jours.

Le traitement par réduction de substrat, miglustat (Zavesca) diminue l'accumulation du glucosylcéramide en excès.

L'efficacité de ce traitement administré par voie orale est moins importante que le traitement enzymatique, et les effets secondaires plus fréquents ; c'est donc un traitement de seconde intention.

\section{Surveillance}

Le suivi des patients nécessite, outre le suivi clinique régulier (tous les 3 à 6 mois), un suivi biologique comportant notamment les différents marqueurs (tous les 6 à 12 mois), un suivi radiologique osseux (IRM) tous les 6 mois au début, puis tous les 24 mois après stabilisation, et abdominal tous les 12 à 24 mois (échographie, IRM), une échographie cardiaque initiale et tous les ans en cas d'atteinte cardiaque.

\section{PRONOSTIC}

Le pronostic vital de la maladie de Gaucher de type 1 n'est en général plus engagé depuis l'utilisation du traitement enzymatique substitutif ; cependant, le pronostic fonctionnel est parfois médiocre compte tenu des possibles séquelles liées à l'atteinte osseuse et des pathologies associées à la maladie (myélome, lymphome, syndrome parkinsonien).

Déclaration d'intérêts : les auteurs ne déclarent aucun conflit d'intérêt en rapport avec cet article.

\section{RÉFÉRENCES}

1. Grabowski GA. Phenotype, diagnosis, and treatment of Gaucher's disease. Lancet 2008;372:1263-71.

2. Grabowski GA. Recent clinical progress in Gaucher disease. Curr Opin Pediatr 2005;17:519-24.

3. Charrow J, Andersson HC, Kaplan P, et al. The Gaucher registry: demographics and disease characteristics of 1698 patients with Gaucher disease. Arch Intern Med 2000; 160: 2835-43

4. Erikson A, Bembi B, Schiffmann R. Neuronopathic forms of Gaucher's disease. Baillieres Clin Haematol 1997 ; 10(4):711-23.

5. Mignot C, Gelot A, Bessieres B, Daffos F, Voyer M, Menez F, et al. Perinatallethal Gaucher disease. Am J Med Genet A 2003 ; 120 (3) : 338-44.

6. Mignot C. Aspects cliniques des formes neurologiques précoces de maladie de Gaucher. Rev Med Interne 2006 ; (Suppl 1):S14-7.

7. Stirnemann J, Caubel I, Kettaneh A, Fain O, Belmatoug N. Aspects épidémiologiques, cliniques, biologiques et thérapeutiques dans la maladie de Gaucher. Presse Med 2003;32 (11):503-11.

8. Barton NW, Brady RO, Dambrosia JM, et al. Replacement therapy for inherited enzyme deficiency-macrophage-targeted glucocerebrosidase for Gaucher's disease. N Engl J Med. 1991;324:1464-70.

Cet article a été publié dans le « Batna Journal of Medical Sciences » BJMS, l'organe officiel de "I'association de la Recherche Pharmaceutique - Batna "

Le contenu de la Revue est ouvert « Open Access » et permet au lecteur de télécharger, d'utiliser le contenu dans un but personnel ou d'enseignement, sans demander l'autorisation de l'éditeur/auteur.

Avantages à publier dans BJMS :

- Open access : une fois publié, votre article est disponible gratuitement au téléchargement

- Soumission gratuite : pas de frais de soumission, contrairement à la plupart des revues « Open Access"

- Possibilité de publier dans 3 langues : français, anglais, arabe

- Qualité de la relecture : des relecteurs/reviewers indépendants géographiquement, respectant l'anonymat, pour garantir la neutralité et la qualité des manuscrits.

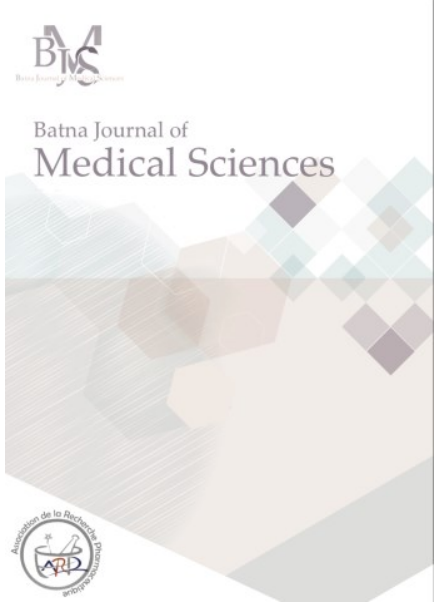

